Damien Brun

Université TÉLUQ

Montréal, QC, Canada

brund@acm.org

Sébastien George

LIUM - EA 4023

Le Mans Université

72085 Le Mans, France

sebastien.george@univ-

lemans.fr

\title{
Keycube: Text Entry Evaluation with a Cubic Device
}

Charles Gouin-Vallerand

Université de Sherbrooke

Sherbrooke, QC, Canada

charles.gouin-

vallerand@usherbrooke.ca

\begin{abstract}
The keycube is a tangible cubic device including a text entry interface for different apparatuses such as augmented, mixed or virtual reality headsets, as well as smart TVs, desktop computers, laptops, tablets. The keycube comprises 80 keys equally disposed on 5 faces. In this paper we investigate keycube text entry performances and the potential typing skill transfer from traditional keyboard. Using prototype implementations, we conducted a user study comparing different cubic layouts and included a baseline from traditional keyboards. Experiments show that users are able to attain about 19 words per minute within one hundred minutes of practice with a QWERTY-based cubic layout, more than twice the speed of an unknown-based cubic layout with similar error rate, and about $30 \%$ of their speed with a traditional keyboard.
\end{abstract}

\section{Author Keywords}

text entry; cube; device; evaluation; input speed; keyboard.

\section{CCS Concepts}

-Human-centered computing $\rightarrow$ Text input; User studies; Keyboards;

\section{Introduction}

With the increasing availability of augmented, mixed and virtual reality headsets, their contexts of use are becoming 


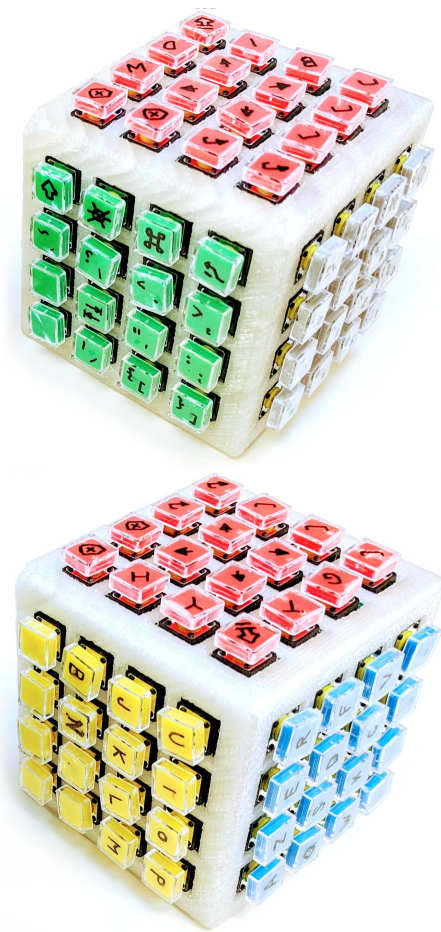

Figure 1: Keycube prototype implementations. diverse but the text entry remain quite ubiquitous no matter the cases. In this regard, several types of interfaces are explored such as a virtual mid-air keyboard [8], speech to text or dedicated devices (e.g. controllers [28], smartphones

[15], gloves [27], rings [23]). However, theses solutions aim to be used for a relatively quick and short text entry. On the other hand, typing is still the most used generic method for long productive text input. Thus, the use of traditional keyboards is also explored [12, 16], offering the advantage of usually being already mastered, but is limited to some situations where users are mostly immobile, seated and requiring a flat surface. To overcome these constraints, we tested the keycube [4] (Figure 1), which is a text entry approach that works by disposing multiple keys on a cubic tangible device. The keycube aims to offer the same flexibility (large quantity and size of keys as well as eye-free and multiple fingers ability) and tactile feedback as the traditional keyboard adopted for long text entry while bringing mobility benefits and more freedom of postures.

With a new generic and interactive device as the keycube, the challenge is to determine which dimensions to consider first for an evaluation. We identified that the most important questions to ask at this stage are: (1) whether the idea of putting keys on a cubic shape device is a reasonable text entry interface; and (2) does the prototype benefits from any skills transfer from a regular keyboard. Therefore, we conducted a user study to determine the following variables upon initial exposure to the device: input speed, error rate and keystrokes per character. Participants were divided into three groups with different cubic layouts, two supposed to benefit from the knowledge transfer and another unfamiliar to the participants. To extend comparative evaluation we included the traditional keyboard as a baseline condition. Results showed that the keycube benefits from exploiting a known-based cubic layout. Participants could achieve 19 words per minute (wpm), twice the speed of an unknownbased cubic layout and more than $30 \%$ of the speed with a traditional keyboard within only 100 minutes practice.

\section{Related Work}

From a text entry perspective, the keycube shares characteristics with many previous works either for their context of use or their form factor and ergonomics. Among them, we limited the scope of related works to typing devices and techniques with physical keys, thus excluding, inter alia, on-screen and mid-air keyboards, speech-to-text interfaces.

The traditional keyboard has been modified in different ways to offer various advantages. For instance, comfort, muscular stress and strain injuries were addressed with split and vertically oriented keyboards [24, 26] without decreasing much of input speed after a week of use. Split and orientation tricks have been used with the RearType technique [25] by disposing keys on the reverse side of a tablet. Exploiting potential knowledge transfer from the QWERTY layout, users achieved $15 \mathrm{wpm}$ within one hour of training. Similar results regarding input speed were achieved with the Back Keyboard [14] where physical size-reduced keys were installed on the backside of a mobile phone. Reducing the overall size of traditional keyboards, including the keys, is also known as compact or mini keyboards, studies reported theoretically [20] and empirically [6] that users may achieve a rate of $60 \mathrm{wpm}$. These devices are used equivalently to smartphones and despite bringing high mobility they are hard to use eye-free (without significant decrease of performances) [7] and they are mostly used with thumbs only, thus are prone to fatigue and muscular stress ('texting thumb' pain) $[5,13]$, in particular for long productive works. Compactness, along with providing access to disabled users, has been explored by Half-QWERTY [22], a one-handed typing technique designed to facilitate skill 


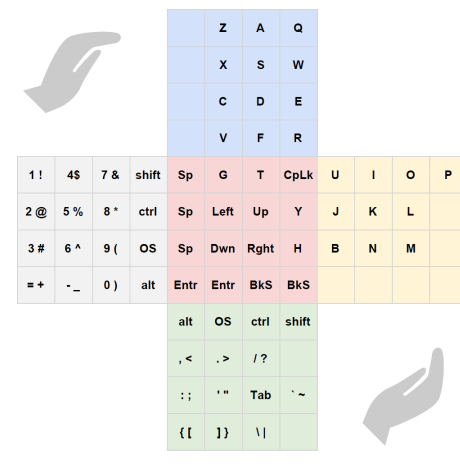

Figure 2: Two-dimensional QWERTY-based cubic layout.

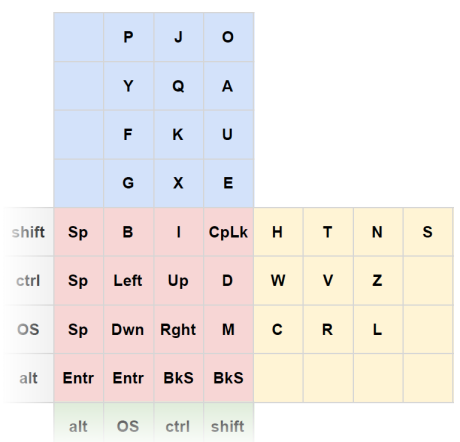

Figure 3: Two-dimensional Unknown-based cubic layout. transfer from two-handed typing. It was shown that QWERTY typists could achieve more than $40 \mathrm{wpm}$ in less than 10 hours. For similar motivations, the Stick Keyboard [11] reduces the physical space requirement and capitalizes on user knowledge of traditional keyboards by mapping four rows of characters to a single row. Users achieved up to $22.5 \mathrm{wpm}$ with a lexicon-based disambiguation technique, but $10.4 \mathrm{wpm}$ by multi-tapping. Other works have explored typing techniques based on highly reduced number of keys (from 1 to 5) [18] but came with a decreased input speed of around 5 to $10 \mathrm{wpm}$ and often implied the need of software optimization and disambiguation. Chording Keyboards allow typing with a very limited number of keys while achieving high input speed. Typing is done by combining multiple keys and may be done either with one or two hands [10]. Studies report on users achieving $35 \mathrm{wpm}$ after 20 hours of practice. The Twiddler [17] is a mobile one-handed chording keyboard with comparable results but suffers from extensive training requirements with substantially low novice performance (3 wpm with $17 \%$ error rate) in virtual reality [3, 9], discouraging users to engage with this solution.

\section{User Study}

The goal of this study was two-fold: (1) upon initial exposure, verify that the keycube is a reasonable device for text entry; (2) verify if the keycube prototype could benefit from the knowledge transfer of traditional keyboards.

\section{Study Design}

We designed a between-subject experiment ( 3 groups of 8 participants) with the cubic layout as an independent variable on 3 levels assigned by group: QWERTY, AZERTY and Unknown. The dependent variables were input speed (words per minute), error rate and keystrokes per character. Furthermore, to extend comparative evaluations, we include the traditional keyboard as a baseline condition.

\section{Participants}

A total of 24 participants (4 left-handed; 15 women; 1936 years old, mean 28) have been recruited mostly from university campus with posters and by word of mouth. Selected participants used a traditional keyboard every day with either QWERTY $(n=12,50 \%)$ or AZERTY $(n=12)$, and were ignorant of the DVORAK layout. To be sure that participants knew their respective layout, we required them to a minimum of 30 words per minute, still allowing a wide range of typing skills. Three of them reported to type without watching the keys. We required participants to be able to read, write and understand English language. All participants had given their written consent and received $35 \mathrm{CAD}$ or 24 EUR as compensation for their participation.

\section{Materials}

Three keycube prototypes were built following the keycube concept [4]. A hand wrist and neck strap were added to the structure for safety and to easily free up the hands. The touch screen was replaced by a 3D printed flat surface, decreasing the overall weight to 383 grams. All keycubes were connected via Bluetooth serial communication at 9600 baud to the same 15-inch laptop. The traditional QWERTY and AZERTY keyboards used for text entry baseline were Apple's keyboards with low profile keys. A program made with Processing (shown in Figure 4) presented English phrases (18 points monospaced font) from the MacKenzie and Soukoreff phrases set [21] and logged key presses with timestamps. The few instances of uppercase characters were lowered. For comparative purposes, the key covers with characters inscriptions were disposed on each keycube following three different cubic layouts. One was defined as the QWERTY-based cubic layout and is presented in the Figure 2. Undefined keys were covered with fully transparent key covers to avoid physical unbalance. An adaptation of the QWERTY-based layout was made to pro- 


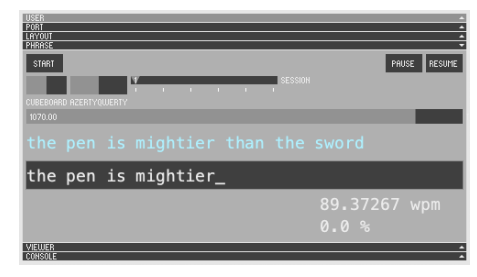

Figure 4: Experiment software.

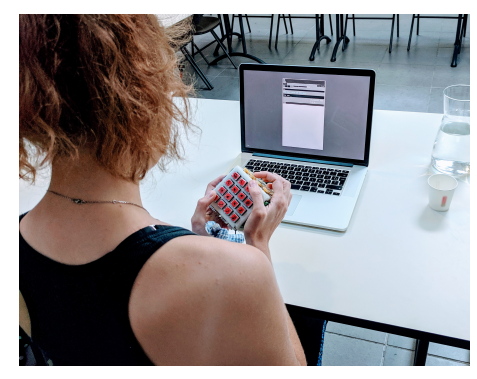

Figure 5: Experiment setup. vide the AZERTY-based layout also supposedly familiar to participants. Only, the keys $\mathrm{Q}$ and $\mathrm{A}, \mathrm{W}$ and $\mathrm{Z}, \mathrm{M}$ and the empty key right of $L$ were switched. On the other hand, the third and Unknown-based cubic layout was to avoid potential benefits from participants previous knowledge. Instead of simply randomizing the assignment of characters, the cubic layout (shown in Figure 3) was based (home and top row swapped) on the DSK (Dvorak Simplified Keyboard) layout optimized for English language [1]. Among the three cubic layouts, only the letter placement was changed because none of the special characters except Space, Enter and Backspace would be involved with the phrases set.

\section{Procedure}

The experiment has been approved by the first author's institution's Ethics Review Committee. After presenting the purpose of the study to the participants, we asked them to take a seat next to a laptop and sign a consent form (Figure 5). We first asked them to fill a pre-study questionnaire on demographics and typing skills, followed by hand measurements. The participants had to read and type a couple of phrases from the set in order to briefly check their current typing speed and understanding of the English language.

Then, one of the three keycubes was assigned to them depending on their traditional keyboard layout usage and their typing speed. Due to similitude of letters placement among the QWERTY and AZERTY layout, we did not expect difference of traditional keyboard skills. Therefore, for the Unknown-based group, half of the participants used regularly a QWERTY layout and the other half an AZERTY layout. The instructor demonstrated how to hold the device, but the participants were allowed to support it however they wished. The instructor showed the needed keys for the experiment (only letters, Space, Backspace and Enter keys), without giving any explanations of the layout. Participants were told to avoid as much as possible pressing unused keys (e.g. white and green keys) and were specifically instructed to "enter the text as quickly and accurately as possible" and started a warm-up round by typing the alphabet twice. To ease practicing, the experiment was composed of 6 typing sessions of 20 minutes each.

The sessions were done consecutively grouped by two and we included short (SB) and long breaks (LB) as such: S1 (session 1), SB, S2, LB, S3, SB, S4, LB, S5, SB, S6. Short break lasted around 1 minute and long break around 30 minutes (at least 20 minutes and at most 80 minutes). Participants were instructed to not use a traditional keyboard during breaks but were allowed to use soft keyboards (e.g. smartphone). The first 5 sessions were performed with the keycube. The last session was performed with a traditional keyboard and participants had to type the alphabet twice in addition to a couple of phrases as warm-up to decrease potential bias due to keycube use. For each participant, the whole experiment was conducted during the same day, therefore we assumed that one session (20 minutes) was enough for assessing the traditional keyboard baseline.

Each session was run similarly and consisted of randomly selected English phrases to be typed by the participants.

The typing speed and error rate were calculated and shown on the software after validating each phrase (Figure 4, bottom right). The experiment lasted around 3 hours and 50 minutes in total, including the breaks.

\section{Results}

Across all participants, we recorded 153,450 key presses and 4,822 phrases typed with the keycube. The first four session were training, we report the results from the keycube final session and traditional keyboard. We removed phrases (about $2.05 \%$ ) with an error rate above $20 \%$ to exclude outliers because they were caused by premature 


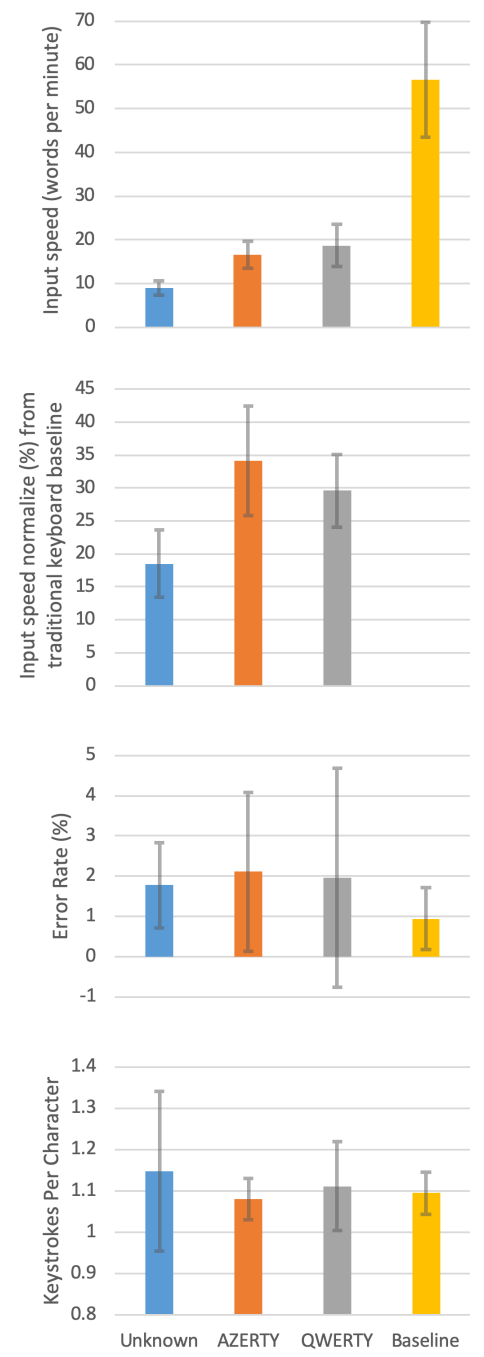

Figure 6: Input speed, error rate and KSPC for each cubic layout and traditional keyboard. validation (e.g. pressing the Enter key twice or too soon). We logged every unintentional key press on unused keys (e.g. white and green faces of the keycube). Participants reported pressing few keys unintentionally mostly with their hand palms. This issue happened in $2.53 \%$ among all users and may have consequences on the input speed by forcing users to be aware and to use the device more sensitively.

INPUT SPEED

The input speed is calculated in Words per Minute as

$$
W P M=\frac{|T|-1}{S} \times 60 \times \frac{1}{5}
$$

where $\mathrm{T}$ is the transcribed text and $\mathrm{S}$ is the time in seconds calculated from the first to the last character pressed. A word is defined as five characters including spaces.

The input speed per layout is reported in the Figure 6 (top) as words per minute and normalized (\%) from the traditional keyboard baseline. The traditional keyboard baseline was expected to receive higher input speed (mean=56.6, $S D=13.1$ ) due to participants' extensive familiarity (years of experience). An analysis of variance (ANOVA) showed that the cubic layout factor had a significant effect on the input speed $\left(F_{2,21}=18.134, p<.0001\right)$. The average typing speed for Unknown-based (mean=9.0, $S D=1.6$ ) was respectively $52 \%$ and $48 \%$ significantly slower than the QWERTY-based (mean=18.7, SD=4.8) and AZERTY-based (mean=16.6, SD=3.0). A post-hoc analysis using Bonferroni pairwise t-tests was performed to determine no significant effects on input speed between the QWERTY-based and AZERTY-based cubic layouts $(p=.298)$.

KEYSTROKES PER CHARACTER AND ERROR RATE

The keystrokes per character (KSPC) is the ratio of the length of input stream (which includes Backspace) to the length of the transcribed text. As a traditional keyboard, the keycube is unambiguous because each letter has a dedicated key, thus for lower case texts, the best KSPC is 1.0. We used the updated version of MacKenzie and Soukoreff error rate [19] calculated as

$$
E R=\frac{M S D(A, B)}{\bar{S}_{A}} \times 100 \%
$$

where $M S D(A, B)$ is the minimum string distance between the presented text $A$ and the transcribed text $B$, and $\overline{S_{A}}$ is the mean size of alignments.

The error rate and KSPC per layout are presented in Figure 6 (bottom). An ANOVA revealed that the cubic layout factor had no significant effect on the Error Rate $(p=0.948)$. All participants across each group seemed to have adopted the same behavior of correcting most of the errors. There was no significant effect either on the KSPC between the cubic layouts $(p=0.599)$. Participants seemed to have avoided as much as possible pressing the wrong keys.

\section{Discussion}

In accordance with the practices of the domain [2], for the integrity of comparisons, the procedure followed was inspired in particular by those used for evaluating the chording keyboard Twiddler [17].

Despite a reported $(n=14)$ bouncing issue from the prototypes that caused unintentional doubling key presses (thus decreasing users' performance), our results show that the keycube with its known-based cubic layouts is already a practical text entry device. Users achieved up to 19 words per minute after practicing 5 sessions of 20 minutes, which corresponded to more than $30 \%$ of their speed with a traditional keyboard. These results are particularly encouraging for such unconventional typing interface in fact users $(n=6)$ openly commented "it is easier than what I thought." 


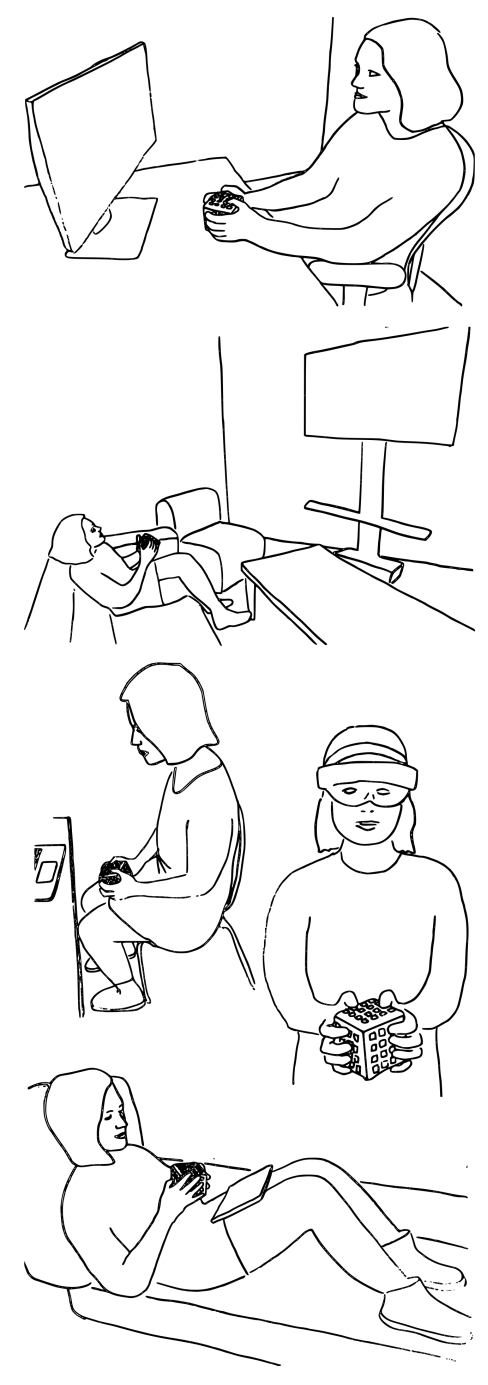

Figure 7: Different home and office work use cases.
The keycube is well positioned in comparison to other devices sharing similar characteristics (supporting mobility, multiple fingers use with large keys and eye-free ability). With comparable error rate, our participants (without instruction) were twice quicker than Twiddler users to attain $19 \mathrm{wpm}$ and four times quicker to attain a normalize input speed at about 30\%, addressing Twiddler main weakness: long learning requirements. With similar practice time, our absolute input speed results are comparable to other solutions that capitalized on the QWERTY keyboard such as RearType and Half-QWERTY. However, their participants were expert and skilled touch typists, thus the RearType had a slower normalized input speed (21\%). Moreover, both of them used some tutorial technics to quickly increase skill acquisition and their results. The input speed is not the only important characteristics for a typing device. Participants $(n=5)$ commented that thanks to the mobility and freedom of postures, it was easier to adopt relaxed positions while typing (e.g. back against the chair, arms along the body, resting on the thighs). In this regard, the keycube could follow the unusual history of the split keyboard design adoption [24] improving health above productivity.

\section{Use cases}

The keycube aims to be used for productive works requiring extensive text entry (e.g. writing letters, reporting, coding) in a ubiquitous computing era with users fully beneficing from their environment at home or in the office. We envision users navigating between different devices and postures, typing seamlessly as much on a regular desktop computer than on smart TV or augmented reality headset (Figure 7).

\section{Future Work}

Besides updates to fix double key-presses (adding a debouncing system), we are planning other studies. We limited the keycube usage to alphabetical letters for internal validity, further investigations including numbers and special characters remain to be done for a better generalization. The instructor observed different behavior (such as typing with multiple fingers or without looking at the device) that need to be quantitatively measured and studied. Beyond initial exposure, longitudinal studies (over weeks) will be conducted to determine expert performance. The experimental setup to desktop configuration with seated participants was still representative to the main use cases of computer consumption and most participants $(n=18)$ hold the device without using the table at all with arms slightly bent (Figure 5), still benefiting mobility. Nonetheless, we plan to investigate different mobile contexts, switching participants' activities, postures (e.g. being stand up) and coupled devices (e.g. with an augmented reality headset).

\section{Conclusion}

With the keycube placing 80 keys onto a cubic shape, we investigated an unconventional typing interface for post and peri-desktop interactions. Our results have shown that users benefit from a knowledge transfer advantage by adopting a QWERTY-based cubic layout. Finally, by having integrated a traditional keyboard baseline, we have shown that a cubic device is already a reasonable and promising typing interface. Users achieved up to $19 \mathrm{wpm}$ in less than two hours, representing more than $30 \%$ of their input speed with traditional keyboard. These preliminary results lead to new possibilities for human-computer interfaces. Since typing is an important generic text entry interface, the keycube could bring adherence to the cube shape and by combination, increase relevance to many previous and future works taking advantage of this platonic solid.

\section{Acknowledgements}

We thank the participants from the user study and all publication support who wrote and provided helpful comments. 


\section{REFERENCES}

[1] Allison M Anderson, Gary A Mirka, Sharon M B Joines, and David B Kaber. 2009. Analysis of alternative keyboards using learning curves. Human Factors 51, 1 (Feb. 2009), 35-45. DOI : http://dx.doi.org/10.1177/0018720808329844

[2] Ahmed Sabbir Arif and Wolfgang Stuerzlinger. 2009 Analysis of text entry performance metrics. In 2009 IEEE Toronto International Conference Science and Technology for Humanity. IEEE, 100-105. DOI : http://dx.doi.org/10.1109/TIC-STH. 2009.5444533

[3] Doug A. Bowman, Christopher J. Rhoton, and Marcio S. Pinho. 2002. Text input techniques for immersive virtual environments: An empirical comparison. In Proceedings of the human factors and ergonomics society annual meeting, Vol. 46. SAGE Publications Sage CA, 2154-2158. DOI : http://dx.doi.org/10.1177/154193120204602611

[4] Damien Brun, Charles Gouin-Vallerand, and Sébastien George. 2019. Keycube is a Kind of Keyboard (K3). In Extended Abstracts of the $2019 \mathrm{CHI}$ Conference on Human Factors in Computing Systems (CHI EA '19). ACM, New York, NY, USA, INT034:1-INT034:4. DOI : http://dx.doi.org/10.1145/3290607.3313258

[5] Joonho Chang, Bori Choi, Amir Tjolleng, and Kihyo Jung. 2017. Effects of button position on a soft keyboard: Muscle activity, touch time, and discomfort in two-thumb text entry. Applied Ergonomics 60 (April 2017), 282-292. DOI :

http://dx.doi.org/10.1016/j. apergo.2016.12.008

[6] Edward Clarkson, James Clawson, Kent Lyons, and Thad Starner. 2005. An empirical study of typing rates on mini-QWERTY keyboards. In CHI'05 extended abstracts on Human factors in computing systems. ACM, 1288-1291. DOI :

http://dx.doi.org/10.1145/1056808.1056898

[7] James Clawson, Kent Lyons, Thad Starner, and Edward Clarkson. 2005. The impacts of limited visual feedback on mobile text entry for the twiddler and mini-qwerty keyboards. In Ninth IEEE International Symposium on Wearable Computers (ISWC'05). IEEE, 170-177. DOI :

http://dx.doi.org/10.1109/ISWC.2005.49

[8] John J. Dudley, Keith Vertanen, and Per Ola Kristensson. 2018. Fast and Precise Touch-Based Text Entry for Head-Mounted Augmented Reality with Variable Occlusion. ACM Trans. Comput.-Hum. Interact. 25, 6 (Dec. 2018), 30:1-30:40. DOI : http://dx.doi.org/10.1145/3232163

[9] Gabriel González, José P. Molina, Arturo S. García, Diego Martínez, and Pascual González. 2009. Evaluation of text input techniques in immersive virtual environments. In New Trends on Human-Computer Interaction. Springer, 109-118.

[10] D. Gopher and D. Raij. 1988. Typing with a two-hand chord keyboard: will the QWERTY become obsolete? IEEE Transactions on Systems, Man, and Cybernetics 18, 4 (July 1988), 601-609. DOI : http://dx.doi.org/10.1109/21.17378

[11] Nathan Green, Jan Kruger, Chirag Faldu, and Robert St. Amant. 2004. A Reduced QWERTY Keyboard for Mobile Text Entry. In CHI '04 Extended Abstracts on Human Factors in Computing Systems (CHI EA '04). ACM, New York, NY, USA, 1429-1432. DOI : http://dx.doi.org/10.1145/985921.986082 
[12] Jens Grubert, Lukas Witzani, Eyal Ofek, Michel Pahud, Matthias Kranz, and Per Ola Kristensson. 2018. Text entry in immersive head-mounted display-based virtual reality using standard keyboards. In 2018 IEEE Conference on Virtual Reality and 3D User Interfaces (VR). IEEE, 159-166. DOI: http://dx.doi.org/10.1109/VR.2018.8446059

[13] Ewa Gustafsson, Sara Thomée, Anna Grimby-Ekman, and Mats Hagberg. 2017. Texting on mobile phones and musculoskeletal disorders in young adults: a five-year cohort study. Applied ergonomics 58 (2017), 208-214. DOI

http://dx.doi.org/10.1016/j . apergo. 2016.06.012

[14] Hwan Kim, Yea-kyung Row, and Geehyuk Lee. 2012 Back Keyboard: A Physical Keyboard on Backside of Mobile Phone Using Qwerty. In CHI '12 Extended Abstracts on Human Factors in Computing Systems (CHI EA '12). ACM, New York, NY, USA, 1583-1588. DOI : http://dx.doi.org/10.1145/2212776.2223676

[15] Youngwon R. Kim and Gerard J. Kim. 2016. Hovr-type Smartphone as a typing interface in vr using hovering. In Proceedings of the 22nd ACM Conference on Virtual Reality Software and Technology. ACM, 333-334. DOI :

http://dx.doi.org/10.1145/2993369.2996330

[16] Pascal Knierim, Valentin Schwind, Anna Maria Feit, Florian Nieuwenhuizen, and Niels Henze. 2018. Physical Keyboards in Virtual Reality: Analysis of Typing Performance and Effects of Avatar Hands. In Proceedings of the $2018 \mathrm{CHI}$ Conference on Human Factors in Computing Systems (CHI '18). ACM, New
York, NY, USA, 345:1-345:9. DOI : http://dx.doi.org/10.1145/3173574.3173919

[17] Kent Lyons, Thad Starner, Daniel Plaisted, James Fusia, Amanda Lyons, Aaron Drew, and E. W. Looney. 2004. Twiddler Typing: One-handed Chording Text Entry for Mobile Phones. In Proceedings of the SIGCHI Conference on Human Factors in Computing Systems (CHI '04). ACM, New York, NY, USA, 671-678. DOI :

http://dx.doi.org/10.1145/985692.985777

[18] I. Scott MacKenzie. 2009. The One-key Challenge: Searching for a Fast One-key Text Entry Method. In Proceedings of the 11th International ACM SIGACCESS Conference on Computers and Accessibility (Assets '09). ACM, New York, NY, USA, 91-98. DOI :

http://dx.doi.org/10.1145/1639642.1639660

[19] I. Scott MacKenzie and R. William Soukoreff. 2002a. A character-level error analysis technique for evaluating text entry methods. In NordiCHI, Vol. 2. 243-246. DOI : http://dx.doi.org/10.1145/572020.572056

[20] I. Scott MacKenzie and R. William Soukoreff. 2002b. A model of two-thumb text entry. In Graphics Interface. 117-124. DOI :

http://dx.doi.org/10.20380/GI2002.14

[21] I. Scott MacKenzie and R. William Soukoreff. 2003. Phrase Sets for Evaluating Text Entry Techniques. In $\mathrm{CHI}$ '03 Extended Abstracts on Human Factors in Computing Systems (CHI EA '03). ACM, New York, NY, USA, 754-755. DOI :

http://dx.doi.org/10.1145/765891.765971 
[22] Edgar Matias, I. Scott MacKenzie, and William Buxton. 1993. Half-QWERTY: A one-handed keyboard facilitating skill transfer from QWERTY. In Proceedings of the INTERACT'93 and CHI'93 Conference on Human Factors in Computing Systems. Citeseer, 88-94. DOI :

http://dx.doi.org/10.1145/169059.169097

[23] Shahriar Nirjon, Jeremy Gummeson, Dan Gelb, and Kyu-Han Kim. 2015. TypingRing: A Wearable Ring Platform for Text Input. In Proceedings of the 13th Annual International Conference on Mobile Systems, Applications, and Services (MobiSys '15). ACM, New York, NY, USA, 227-239. DOI :

http://dx.doi.org/10.1145/2742647.2742665

[24] David Rempel. 2008. The split keyboard: An ergonomics success story. Human Factors 50, 3 (2008), 385-392. DOI :

http://dx.doi.org/10.1518/001872008x312215

[25] James Scott, Shahram Izadi, Leila Sadat Rezai, Dominika Ruszkowski, Xiaojun Bi, and Ravin Balakrishnan. 2010. RearType: text entry using keys on the back of a device. In Proceedings of the 12th international conference on Human computer interaction with mobile devices and services. ACM, 171-180. DOI :

http://dx.doi.org/10.1145/1851600.1851630

[26] Gerard P. Van Galen, Hanneke Liesker, and Ab de Haan. 2007. Effects of a vertical keyboard design on typing performance, user comfort and muscle tension. Applied ergonomics 38, 1 (2007), 99-107. DOI: http://dx.doi.org/10.1016/j. apergo. 2005.09.005

[27] Eric Whitmire, Mohit Jain, Divye Jain, Greg Nelson, Ravi Karkar, Shwetak Patel, and Mayank Goel. 2017. DigiTouch: Reconfigurable Thumb-to-Finger Input and Text Entry on Head-mounted Displays. Proc. ACM Interact. Mob. Wearable Ubiquitous Technol. 1, 3 (Sept. 2017), 113:1-113:21. DOI : http://dx. doi.org/10.1145/3130978

[28] Andrew D. Wilson and Maneesh Agrawala. 2006. Text entry using a dual joystick game controller. In Proceedings of the SIGCHI conference on Human Factors in computing systems. ACM, 475-478. DOI : http://dx.doi.org/10.1145/1124772.1124844 